\title{
Chronic Obstructive Pulmonary Disease Exacerbations: Current Controversies
}

\author{
Shawn D. Aaron, MD
}

The Ottawa Hospital Research Institute, University of Ottawa, Ottawa, Ontario, Canada

\section{ABSTRACT}

Exacerbations of chronic obstructive pulmonary disease (COPD) are characterised by acute worsening of dyspnoea, sometimes accompanied by cough and sputum production and increased sputum purulence. Exacerbations carry with them important clinical and economic consequences including lost work productivity, increased utilisation of health care resources, temporary or permanent reductions in lung function and exercise capacity, hospitalisation, and sometimes death. Significant unresolved controversies surround COPD exacerbations. Controversies can be broadly grouped into the following categories: 1) definition of exacerbations; 2) pathogenesis of exacerbations; 3) prediction of exacerbations and early intervention in individual patients; 4) the role of patient self-management and COPD action plans in treatment of exacerbations; and 5) use of antibiotics to treat outpatient exacerbations. This paper will review the current state of the art with respect to how we define, predict, and treat COPD exacerbations, along with controversies and challenges associated with optimal management of exacerbations. (BRN Rev. 2019;5(4):263-76) Corresponding author: Dr. Shawn Aaron, saaron@ohri.ca

Key words: Antibiotics. Chronic obstructive pulmonary disease. Exacerbations. Selfmanagement. 


\section{INTRODUCTION}

Chronic obstructive pulmonary disease (COPD) is a chronic illness that may be periodically punctuated by acute worsening of symptoms characterised clinically by greater dyspnoea, cough and sputum production, and increased sputum purulence. These acute worsening of symptoms have been termed acute exacerbations of COPD (AECOPD). These exacerbations are common and carry with them important clinical and economic consequences including lost work productivity, acute declines in quality of life, temporary or permanent reductions in lung function and exercise capacity, hospitalisation, and sometimes death ${ }^{1-7}$.

Data from longitudinal studies of COPD cohorts has revealed that COPD exacerbations become more frequent as the severity of COPD progresses, and that a subgroup of patients may be prone to frequent exacerbations ${ }^{8}$. Similarly, data from administrative cohorts suggests that the first severe exacerbation requiring hospitalisation places patients with COPD at increased risk for future severe AECOPD and is associated with an increased risk of all-cause mortality ${ }^{9}$. Given the emerging clinical, economic and research importance of AECOPD, it is critical that exacerbations be rigorously defined and that exacerbation events be easily ascertained and appropriately treated in clinical practice.

The objective of this narrative review will be to discuss the current state of the art with respect to how we define, predict and treat COPD exacerbations. Both mild and moderate exacerbations of COPD that are self-treated or treated in outpatient settings by healthcare practitioners, and severe exacerbations of COPD that require inpatient treatment in hospital will be discussed. This review will explore current controversies around these topics. Controversies have been broadly grouped into the following categories: 1) definition of exacerbations; 2) pathogenesis of exacerbations; 3) prediction of exacerbations and early intervention in individual patients; 4 ) the role of patient self-management and COPD action plans in treatment of exacerbations; and 5) use of antibiotics to treat outpatient exacerbations. Relevant articles for this review were retrieved from the Medline and PubMed databases using the following search terms: "COPD exacerbation", "exacerbations of COPD", and "COPD exacerbation controversies". Abstracts were assessed for potential relevance to the topic and applicable articles were included in this review.

\section{CONTROVERSY \#1- DEFINITION OF EXACERBATIONS}

Despite the best efforts of clinicians and guideline committees, current definitions of exacerbations of COPD are imperfect and fraught with problems. The 2019 Global initiative for Obstructive Lung Disease (GOLD) definition of AECOPD defines an AECOPD as "an acute worsening of respiratory symptoms that results in additional therapy"10. Exacerbations are classified as: 1) "mild" if they are treated with short-acting bronchodilators only; 2) "moderate" if they are treated with short-acting bronchodilators plus antibiotics and/or oral corticosteroids; or 3) "severe" if the patient visits the emergency room or requires hospitalisation because of an exacerbation $^{11}$. The problem with this definition is that "an acute worsening of respiratory symptoms" can be provoked by many conditions 
other than AECOPD. The current definition is nonspecific and a wide variety of cardio-pulmonary disorders, such as pneumonia, bronchiectasis, congestive heart failure, or pulmonary embolism ${ }^{12}$, can potentially mimic symptoms of AECOPD, and hence be mistakenly diagnosed as AECOPD.

Exacerbations can be defined using symptom-based or event-based definitions, or a combination of both. Symptom-based definitions rely on patient-reporting of worsening respiratory symptoms either to a healthcare practitioner or within a symptom diary. Typical symptoms of AECOPD would include worsening dyspnoea, cough, increased sputum volume or increased sputum purulence. In contrast, event-based definitions capture patients whose condition has changed enough to require a change in treatment ${ }^{13}$.

An obvious advantage of using a symptom-based definition is that symptoms are important and that they are the primary concern of the patient. Therefore, defining an exacerbation based on symptoms has clinical relevance to patients and to caregivers. A further advantage is that validated tools to capture symptom-based AECOPD's exist and include patient diary cards ${ }^{14}$ and the EXACerbation of chronic pulmonary disease Tool (EXACT) $)^{15}$.

The disadvantages include the subjective nature of symptom-based definitions. In some cases, it is difficult for the patient and the clinician to decide if a patient's symptoms are "increased" more than usual ${ }^{16}$. In addition, numerous studies have shown that symptom-based AECOPDs often go unreported, leading to an under-estimation of patient exacerbation rates ${ }^{17,18}$. Finally, paper-based diary cards and home-based symptom assessment tools are plagued by poor adherence and recall biases related to delays in entering symptoms (diary hoarding), thus leading to retrospective record entry and reduced data accuracy ${ }^{19}$. Use of electronic diaries, rather than paper-based methods, may allow for daily prompting of patients and may help to alleviate delays in data entry ${ }^{19}$.

Event-based definitions circumvent the problems associated with identifying a group of symptoms by simply capturing patients whose condition has changed enough to require a change in treatment or hospitalisation. Advantages include that event-based definitions capture exacerbations that are more clinically important compared to pure symptom-based definitions. These exacerbations are also associated with direct healthcare costs in the form of additional medications and physician visits or hospitalisations and are therefore useful for economic analy$\operatorname{ses}^{20}$. Disadvantages include that event-based definitions can be confounded by patient socioeconomic status, geography, and access to healthcare resources. Patients with limited access to healthcare resources, whether because of geographic isolation, immobility, or poverty, will necessarily experience fewer events ${ }^{21}$. This issue also confounds measurement and definition of severe exacerbations requiring hospital admission. The decision to admit a patient to hospital to treat a COPD exacerbation can be influenced by multiple subjective elements from both patient and doctor as well as structural elements, including socioeconomic status of the patient, existing patient home support systems, and the health system profile. This coexisting 
controversy imbricates and modulates the problem of variability in defining a severe AECOPD.

\section{Towards an improved definition of acute exacerbations}

Current definitions of exacerbations of COPD are limited by the subjective nature of symptoms used to define their occurrence, the non-specificity of symptoms for pulmonary versus cardiac origin, patient non-reporting of exacerbation events to their healthcare providers, and the variable response of clinicians to the patient's report of symptoms. Within this manuscript, the term AECOPD is used to describe an increase in respiratory symptoms in patients with COPD. The lack of an objective biomarker that indicates the onset of the event as well as its severity and prognosis is a major problem with the current definition of exacerbation. There is a need to develop a new, easy to use, and objective definition of exacerbation that incorporates symptom change with biomarker characterisation. If symptoms of AECOPD could be combined with sensitive and specific biomarkers for AECOPD, then diagnosis would become less subjective and hence more reliable. It is important to note that a variety of different cardiopulmonary disorders can cause worsening of respiratory symptoms in a patient with COPD. If these other cardio-pulmonary disorders (e.g., thromboembolism, pneumonia, acute congestive heart failure) are detected and thought to have primarily caused the patient's acute respiratory deterioration, then this patient would not be considered to be suffering from an AECOPD, and instead be diagnosed as having respiratory decompensation from acute pulmonary embolism, for example. Sometimes this distinction can be difficult, since in many cases it is possible that multiple different mechanisms may act simultaneously to cause dyspnoea in an individual patient with COPD.

Figure 1 depicts a flow diagram describing a suggested clinical approach to suspected COPD exacerbations. The necessary investigations needed to confirm a diagnosis of COPD exacerbation, as well as investigations needed to rule out alternative diagnoses are provided. This new definition and grading system have been adapted and refined from a "precision medicine" proposal advanced by Agusti et al. ${ }^{22}$ and from a paper by Celli et al. ${ }^{23}$ describing grading COPD clinical states. Newer evidence generated by Bafadhel et al. ${ }^{24}$ and Noell et al. ${ }^{25}$ has been incorporated into the definition. According to the new proposed definition, the clinical hallmarks of COPD exacerbation are increased dyspnoea ( $\geq 5$ using a 0-10 scale), sometimes but not necessarily accompanied by increased cough, sputum volume, or sputum purulence. Laboratory evidence necessary to confirm a COPD exacerbation include: oxygen desaturation $\leq 4 \%$ below that of stable state, elevated C-reactive protein (CRP) $(\geq 3 \mathrm{mg} / \mathrm{L})$ and circulating blood neutrophilia ( $\geq 9000$ neutrophils/mm) or circulating blood eosinophilia ( $\geq 2 \%$ blood eosinophils). Other aetiologies of respiratory decompensation should be ruled out by obtaining a chest radiograph to exclude pneumonia or pleural effusion/pneumothorax and by measuring B-type natriuretic peptide (BNP) and troponin to help rule out congestive heart failure or myocardial ischaemia.

Although this proposed definition for COPD exacerbation appears to be more specific than what currently exists, the latter definition for AECOPD and diagnostic approach to AECOPD outlined in figure 1 needs to be validated in prospective clinical studies. 


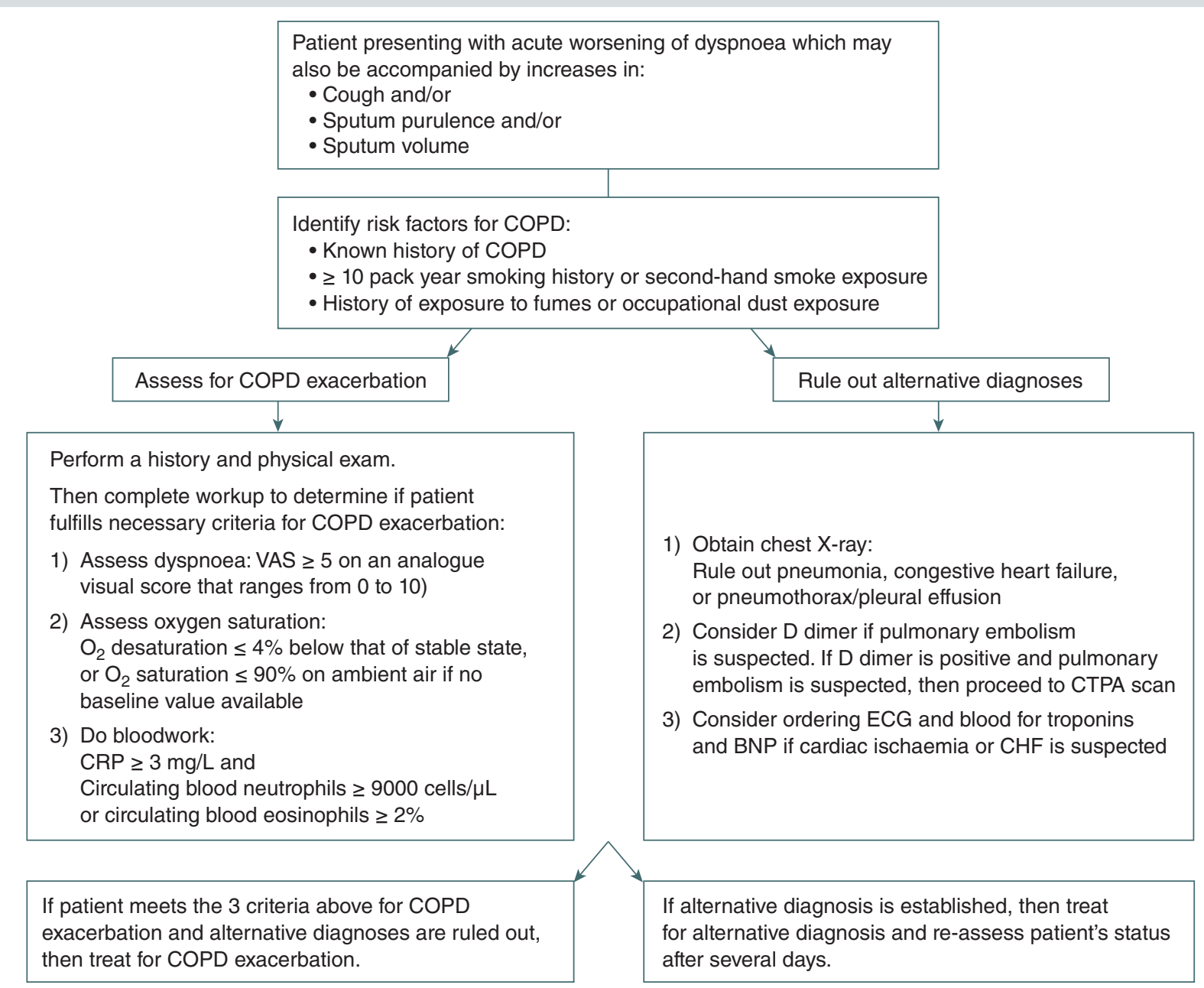

Figure 1. A suggested clinical approach to suspected COPD exacerbations.

Confirmation of COPD exacerbation requires: 1) Symptoms compatible with COPD exacerbation; 2) A patient history of COPD or risk factors for COPD; 3) Laboratory investigations compatible with COPD exacerbation; and 4) Laboratory investigations that rule out other diagnoses. BNP: B-type natriuretic peptide; CHF: congestive heart failure; COPD: chronic obstructive pulmonary disease; CRP: C-reactive protein; CTPA: computed tomography pulmonary angiography; ECG: electrocardiography; VAS: visual analogue scale.

\section{CONTROVERSY \#2- PATHOGENESIS OF EXACERBATIONS}

Determining the aetiology of an exacerbation of COPD is difficult and the topic remains controversial. The exacerbations are caused by complex interactions between the host, respiratory viruses, and airway bacteria, leading to an increase in the inflammatory burden within the airway ${ }^{6,26}$. Studies of COPD exacerbation suggest that airway neutrophils, as well as systemic and local mediators of inflammation including interleukin (IL)-8 and tumour necrosis factor (TNF)-alpha increase during AECOPD compared to the stable state ${ }^{6,27}$. Migration and activation of neutrophils within the airway leads to release of proteases and reactive oxygen species which further damages the airway epithelium and leads to worsening of airway inflammation and airflow limitation (Fig. 2). A minority 


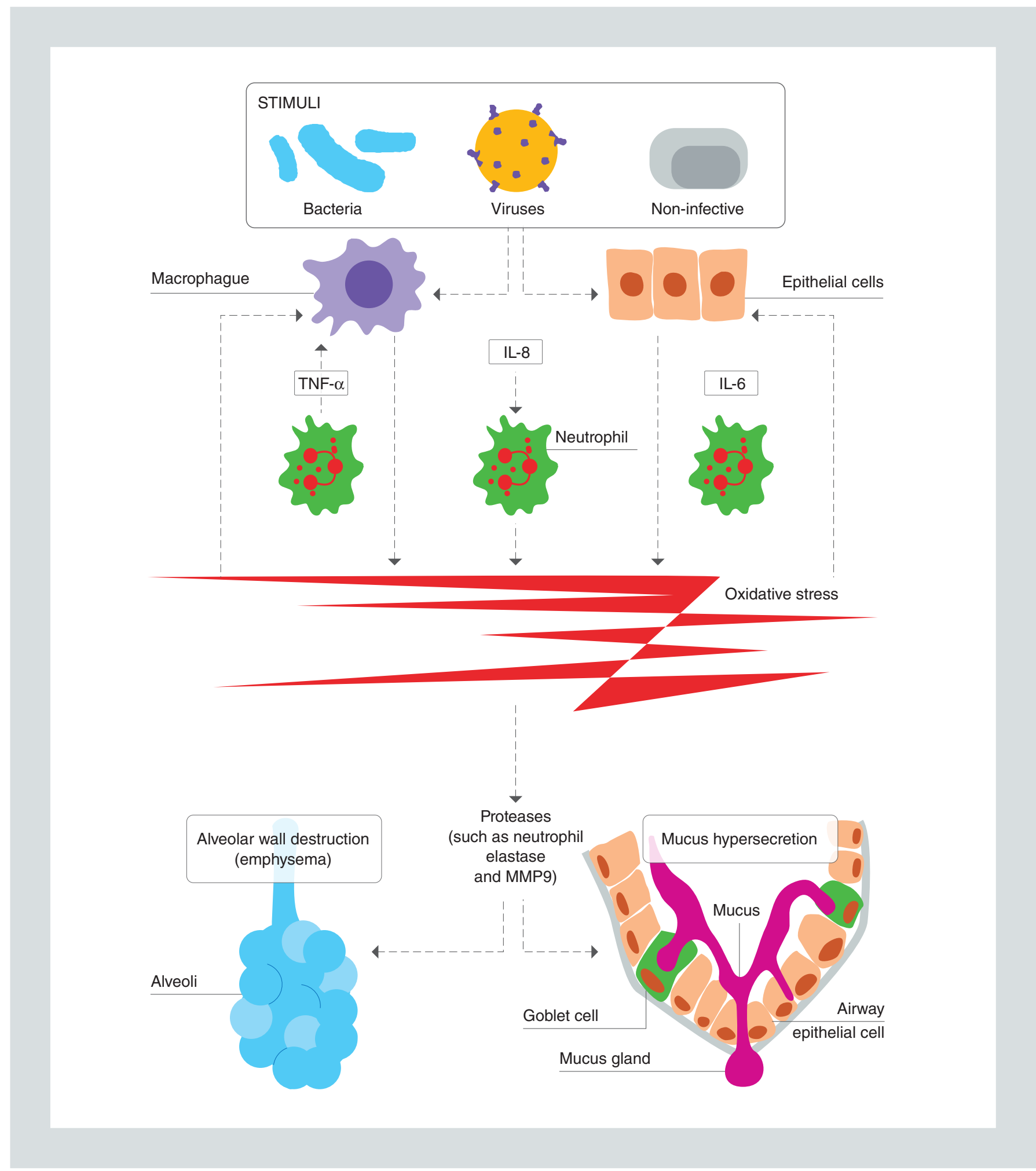

Figure 2. Mechanism for acute exacerbations in chronic obstructive pulmonary disease.

Triggers of acute exacerbations of chronic obstructive pulmonary disease (AECOPD) include infectious agents such as bacteria and viruses and non-infectious agents such as air pollution. These stimuli activate airway epithelial cells and macrophages to release inflammatory cytokines including tumour necrosis factor (TNF)- $\alpha$, interleukin (IL)-6, and IL-8. These cytokines lead to neutrophil recruitment and the release of reactive oxidant species and proteases from activated neutrophils which further magnify the inflammatory process (adapted from Han $M K^{67}$, reprinted with permission of the American Thoracic Society, (C) 2019 American Thoracic Society.). 
of exacerbations are associated with airway and sputum eosinophilia ${ }^{24,28}$.

Exacerbations of COPD are accompanied by changes in airway physiology that induce dyspnoea $^{29}$. A clinical study suggested that patients experience acute worsening of airflow limitation accompanied by acute lung hyperinflation at the time of a moderate $\mathrm{AECOPD}^{30}$. At recovery, 60 days after the initial onset of exacerbation, there was significant improvement in inspiratory capacity and forced expiratory flow in one second $\left(\mathrm{FEV}_{1}\right)$ by $18 \%$ above exacerbation values, accompanied by a decrease in gas trapping (residual volume) by $11 \%$. Improvements in dyspnoea following AECOPD were significantly correlated with reduction in lung hyperinflation and consequent increase in expiratory flow rates, suggesting that dyspnoea during AECOPD is likely partially mediated by dynamic hyperinflation that occurs in response to airflow limitation associated with AECOPD events ${ }^{30}$ (Fig. 3).

In general, viral and bacterial infections are the most important triggers of exacerbations ${ }^{31-33}$. A review of 24 studies of viral infections in COPD exacerbations (from 2001 to 2015) showed virus detection rates in respiratory specimens of $22-64 \%{ }^{34}$. Studies also suggest that AECOPD are often associated with the isolation of new strains of airway bacteria, with an accompanying host inflammatory response to these new strains $^{31,35}$. Respiratory bacteria and viruses often act in combination ${ }^{36}$; studies of experimentally induced rhinovirus infection in patients with COPD suggest that airway bacterial super-infection commonly follows acute rhinovirus infection and that infection with both viruses and bacteria can exert a synergistic inflammatory effect ${ }^{37}$. Co-infection with viruses and bacteria has been detected in $25 \%$ of exacerbations and is associated with more severe functional impairment and longer hospitalisations $^{38}$. Molecular techniques, including multiplex Polymerase Chain Reaction (PCR) have allowed investigators to more sensitively detect acute viral respiratory tract infections causing AECOPD, however in approximately one-third of severe exacerbations a specific infectious or environmental aetiology cannot be identified ${ }^{39}$. In some cases, environmental factors such as air pollution may trigger respiratory deterioration and exacerbation of $\mathrm{COPD}^{40}$.

Investigations using cluster analysis suggest that exacerbations can be grouped into four categories: bacterial, viral, eosinophilic-predominant, and a fourth category associated with limited changes in the inflammatory profile termed "pauciinflammatory"24. Each of these clusters is associated with a biomarker, suggesting that biomarkers may become useful to categorise AECOPD aetiology.

Recent attention has been placed on using alternative biomarkers to phenotype AECOPD. Procalcitonin, the prohormone of calcitonin, is released in different tissues in response to bacterial, but not viral, infections or nonspecific inflammation ${ }^{41}$. For this reason, procalcitonin can theoretically be used as a biomarker to help differentiate between AECOPD caused by bacteria and exacerbations due to other aetiologies $^{42}$. A recent meta-analysis of eight trials evaluating 1062 patients with AECOPD demonstrated that procalcitonin-based protocols could decrease antibiotic prescription for AECOPD (rate ratio [RR] 0.56, 95\% confidence interval [CI] 0.43-0.73) without adversely affecting clinical outcomes such as rate of treatment failure, length of hospitalisation, 
A

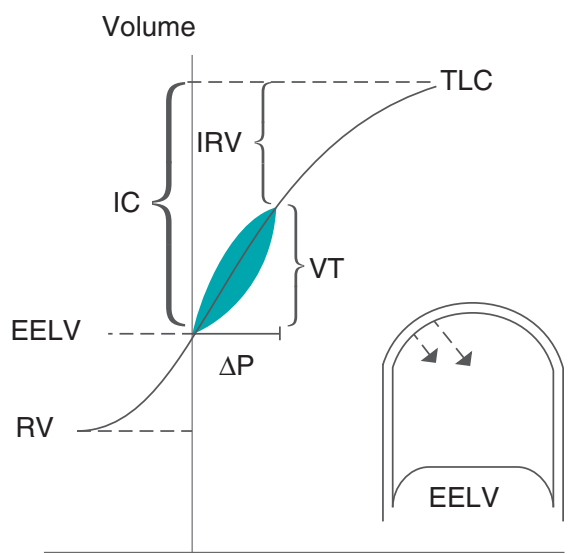

Pressure
B

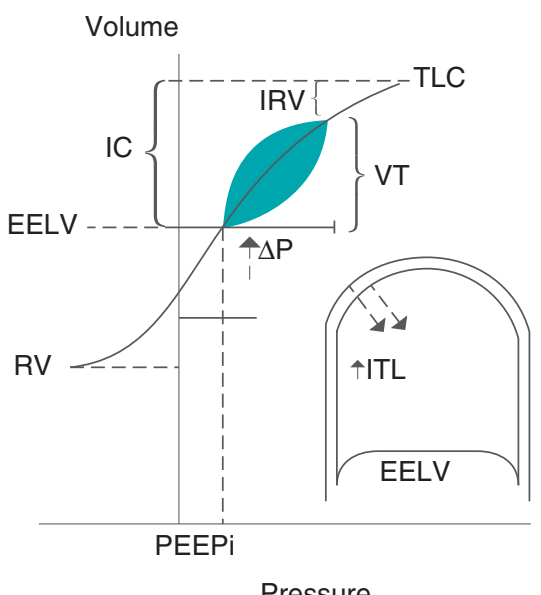

Pressure

FIGURE 3. Schematic diagram of the patho-physiologic effects of chronic obstructive pulmonary disease (COPD) exacerbation.

Pressure-volume plots are shown during a) stable COPD and b) COPD exacerbation. During exacerbation, worsening airflow obstruction leads to dynamic hyperinflation with increased end-expiratory lung volume (EELV) and air trapping manifested as an increased residual volume (RV). Corresponding reductions occur in inspiratory capacity (IC) and inspiratory reserve volume (IRV). As a result, tidal breathing is shifted to the right on the pressure-volume curve, closer to TLC, resulting in unfavourable overall compliance of the respiratory system. Increased mechanical pressures must be generated by the respiratory muscles to maintain tidal volume (VT). At EELV during an exacerbation, intrapulmonary pressures do not return to zero, representing the development of intrinsic positive end expiratory pressure (PEEPi) which imposes further increased inspiratory threshold loading (ITL) on the inspiratory muscles (inset) (reproduced with permission from O'Donnell $D E^{29}$, (c) 2006 Thorax, BMJ Publishing Group Ltd.).

exacerbation recurrence rate or mortality ${ }^{43}$. Similarly, newer technologies such as the electronic nose, which detects volatile organic compounds in exhaled air that are associated with bacterial airway infection, may also help distinguish AECOPD due to bacterial infection from those without infection ${ }^{44}$.

\section{CONTROVERSY \#3- PREDICTION OF EXACERBATIONS AND EARLY INTERVENTION IN INDIVIDUAL PATIENTS}

Prospective studies have shown that the most important risk factor for future exacerbations is a patient history of prior exacerbations ${ }^{45}$. Data from longitudinal studies of COPD cohorts has revealed that $\mathrm{AECOPDs}$ become more frequent as the severity of COPD progresses ${ }^{46}$, and that risk factors for exacerbations include low baseline lung function, increased symptom burden, radiographic evidence of emphysema, and a history of chronic bronchitis ${ }^{8,45,47}$. Similarly, data from administrative cohorts suggests that the first severe exacerbation requiring hospitalisation places patients with COPD at increased risk for future severe AECOPD and is associated with an increased risk of all-cause mortality ${ }^{9,48}$.

Several studies of the "frequent exacerbator" phenotype have been published recently. Although the frequent exacerbator may be responsible for a significant proportion of healthcare utilization in COPD, the true prevalence 
of this phenotype is not clear. In the Evaluation of COPD Longitudinally to Identify Predictive Surrogate Endpoints (ECLIPSE) study, a history of previous exacerbations was the greatest risk factor for future exacerbations ${ }^{45}$. However, of those with two or more exacerbations in the year prior to enrolment, only $58 \%$ had frequent exacerbations in year 1 , and only $60 \%$ of those with frequent exacerbations in year 1 had frequent exacerbations in year 2. Thus, it appears that the phenotype of the "frequent exacerbator" can vary year to year and does not necessarily convey a strong positive predictive value for future events. Recent literature has also revealed that the "frequent exacerbator" phenotype is not quite as common as once believed. In the SubPopulations and InteRmediate Outcome Measures In COPD Study (SPIROMICS) cohort, only 82 of 1105 subjects $(7 \%)$ had at least one exacerbation per year during three years of follow-up, and only $23(2 \%)$ had two or more exacerbations per year ${ }^{8}$.

A topic that deserves further attention is whether we can detect exacerbations early, and whether early detection and treatment can improve outcomes. Studies suggest that prompt therapy of AECOPD is associated with quicker recovery time. Wilkinson et al. ${ }^{49}$ showed that the length of delay (in days) between onset of AECOPD and treatment was associated with longer symptom recovery time back to baseline. Chandra et al. ${ }^{50}$ showed that a delay of $>24$ hours from time of onset of AECOPD symptoms until emergency department (ED) presentation was associated with a 2.3-fold increased risk of need for hospital admission. These observational studies suggest that the earlier we treat AECOPD, the better the clinical outcomes.
A major hurdle to early detection of AECOPDs is that blood and plasma laboratory biomarkers of exacerbation are not sensitive or specific to predict AECOPD ${ }^{6,51}$, and home monitoring of peak expiratory flow rates correlates poorly with event-based exacerbations ${ }^{52}$. The possibility of newer technologies such as activity and heart rate monitors, portable continuous pulse oximeters, and telemedicine as tools to help identify symptoms and signs that herald an impending exacerbation holds promise and is being investigated in multiple studies. However, at present, home measurement of daily symptom scores remains the gold standard to establish an exacerbation event. Studies using home diary card data have defined an exacerbation as the first of two or more consecutive days on which the patient records two or more new or worsening respiratory symptoms ${ }^{53}$. Unfortunately, diary card recording of daily respiratory symptoms may produce false-positive signals. A study by Aaron et al. ${ }^{54}$ showed that $55 \%$ of respiratory deteriorations are transient and do not result in AECOPD. The same study showed that in 1115 of 1995 COPD exacerbations (56\%) recorded using home diary cards the onset of exacerbation was sudden and the exacerbation threshold was crossed on the same day respiratory symptoms began ${ }^{54}$. This suggests that prediction of exacerbation events and institution of early treatment in an outpatient setting may be difficult since in many cases patients do not exhibit gradual warning signs of exacerbation, and they may have difficulty accessing healthcare professionals in a timely fashion once a full-blown exacerbation is upon them. Given this, investigators have concentrated on action plans and home self-management of AECOPD to improve the timeliness of access of patients to AECOPD therapies. 


\section{CONTROVERSY \#4- THE ROLE OF PATIENT SELF-MANAGEMENT AND ACTION PLANS IN TREATMENT OF EXACERBATIONS}

A 2017 Cochrane review ${ }^{55}$ studied 22 clinical trials focused on self-management of AECOPD. Each of the included trials compared interventions that included an action plan for AECOPD against usual care. The review found that selfmanagement intervention strategies in patients with COPD was associated with improved health-related quality of life as measured by the St. George's Respiratory Questionnaire (SGRQ). Over 12 months, there was a statistically significant beneficial effect of self-management interventions with action plans on Health-Related Quality of Life (HRQoL), as measured by the SGRQ total score, with a mean difference from usual care of -2.69 points (95\% CI -4.49 to -0.90 ). Patients assigned to self-management with an action plan had a statistically significant lower risk for at least one respiratory-related hospital admission compared with participants who received usual care (odds ratio [OR] 0.69, 95\% CI 0.51 to 0.94). There was no statistically significant effect observed from self-management on the number of COPD exacerbations and no difference in all-cause mortality observed (risk difference $0.19 \%$, 95\% CI $-2.3 \%$ to $2.6 \%$ ). However, an exploratory analysis showed a very small, but significantly higher respiratory-related mortality rate in the self-management intervention group compared to the usual care group (risk difference $2.8 \%, 95 \%$ CI $0.49 \%$ to $5.1 \%)^{55,56}$.

This exposes the controversy, could AECOPD action plans possibly be dangerous for some patients? A trial by Fan et al. ${ }^{57}$ randomised 426 patients with COPD from Veteran's Affairs
(VA) hospital-based outpatient clinics to either usual care or a self-management intervention consisting of a) an educational programme; b) written action plans and prescriptions for AECOPD management (antibiotics and/or steroids to be used at home); and c) access to case managers by phone ${ }^{57}$. The primary outcome was hospitalisations for COPD. The study was terminated early by the Data Safety Monitoring Board because of excess mortality seen in the intervention group. Although no differences were seen in the time to first hospitalisation for AECOPD, a significant difference in overall survival was seen in favour of the usual care arm (hazard ratio $[\mathrm{HR}]=3.00,95 \%$ CI 1.46 - 6.17). Twenty-eight deaths (17\%) occurred in the comprehensive care management group compared with 10 deaths (7\%) in the usual care group $(\mathrm{p}=0.003)^{57}$.

Why would disease self-management with institution of an AECOPD action plan for home treatment put patients potentially at risk? The first potential risk of self-management action plans is that patients may mistake simple variations in day-to-day symptoms for an exacerbation, and this may result in unnecessary exposure to therapy. A previous study showed that $55 \%$ of respiratory deteriorations are transient and do not result in $\mathrm{AECOPD}^{54}$. If patients overuse antibiotics and oral steroids for transient respiratory deteriorations, then the side effects associated with treatment could cause harm. Results from the Fan study ${ }^{57}$ suggest this could possibly be a factor; patients in the self-management arm had the same rate of AECOPD as those in the usual care arm, however they were statistically more likely to use oral steroids than those in the usual care arm (RR for oral steroid usage $1.25,95 \%$ CI 1.05 to 1.48 ). 
A second potential risk of self-management with action plans is that patients may treat themselves at home and delay seeking medical attention for respiratory symptoms. Delays in seeking medical attention may occur because self-treatment may provide a false sense of security, causing a delay in seeking care for a COPD exacerbation or other comorbid conditions as heart failure. There are data to support the idea that patients in the Fan trial that showed increased mortality did not self-manage appropriately ${ }^{58}$. Only $4.5 \%$ of these patients called in to the care managers before starting treatment for their exacerbation, and the time to initiation of antibiotics or steroids was unsatisfactorily long. The reasons for a higher mortality will likely never be known, but it is possible that these high-risk patients may have needed earlier assessment by a trained professional, or that self-management led to overconfidence and treatment delays ${ }^{58}$.

Finally, it should be noted that the findings from the Fan trial differ from other trials that evaluated similar interventions in similar COPD populations. Bourbeau et al. ${ }^{59}$ conducted a randomised trial of self-management in 181 patients with COPD, all of whom had been hospitalised in the previous year. The intervention included seven to eight weekly educational sessions in the patient's home, an action plan, and a case manager who made monthly calls for a year. During the one-year follow-up, they found a $40 \%$ reduction in COPD hospitalisations in the intervention group, with nine $(9.5 \%)$ deaths in the usual care group and five $(5.2 \%)$ deaths in the intervention group. A similar study by Rice et al..$^{60}$ evaluated self-management in 743 patients with COPD at 5 VA medical centres. As with the Bourbeau trial, the intervention included an action plan and a case manager who made monthly telephone calls for a year. The intervention differed principally in that the initial educational program was condensed to a single 1- to 1.5-hour on-site session. The investigators found a $41 \%$ reduction in the composite primary outcome of COPD-related hospitalisations or ED visits in the intervention group. Forty-eight (12.9\%) deaths occurred in the usual care group versus $36(9.7 \%)$ in the intervention group ${ }^{60}$.

In summary, an integrated COPD self-management strategy comprised of patient education, an action plan, and access to a case manager seems to decrease respiratory related hospitalisations. There do not appear to be any mortality benefits associated with this strategy. Respiratory-related mortality may be slightly higher in the self-management group, possibly because some patients being treated with self-management strategies delay seeking medical attention for respiratory deteriorations.

\section{CONTROVERSY \#5- USE OF ANTIBIOTICS TO TREAT OUTPATIENT EXACERBATIONS}

The role of antibiotics for treatment of moderate exacerbations in the outpatient setting remains controversial. The first trial of antibiotics for outpatient COPD exacerbations was done in 1987 by Anthonisen et al. ${ }^{61}$. This study showed a higher 21-day symptom resolution rate after treatment with doxycycline, co-trimoxazole, or amoxicillin. The beneficial effect of antibiotics was more pronounced in patients with increased dyspnoea, sputum volume, and sputum purulence. Subsequently a trial by Llor et al. ${ }^{62}$, published in 2012, showed 
higher clinical resolution and longer time to next exacerbation with amoxicillin and clavulanate than with placebo. A total of $74 \%$ of patients treated with amoxicillin/clavulanate versus $60 \%$ treated with placebo were considered cured after 9-11 days of treatment (difference, $14.2 \%$; 95\% CI, 3.7-24.3).

In contrast, a recently published trial by van Velzen et al. ${ }^{63}$ used doxycline for seven days to treat outpatient AECOPD and failed to show obvious benefits. All patients in this trial received a 10-day course of oral corticosteroids. Median time to the next exacerbation was 148 days (95\% CI 95-200) in the doxycycline group and 161 days (118-211) in the placebo group $(p=0.91)$. A secondary outcome measure was treatment non-response at day 21, defined as absence of improvement in patient-reported respiratory symptoms, prescription of open-label antibiotics or a new course of oral corticosteroids, admission to hospital for an exacerbation, or death. 32 (21\%) of 150 patients had not responded to doxycycline by day 21 compared with 46 (31\%) of 151 in the placebo group $(p=0.07)^{63}$.

Although the study by van Velzen et al. did not definitively show that treatment non-response was superior in those treated with doxycline, the study was likely underpowered to examine treatment non-response at 21 days as the $p$ value of 0.07 was marginally non-significant. A recent Cochrane systematic review ${ }^{55}$ showed that in outpatients, treatment non-response one month after treatment initiation was not different between patients receiving antibiotics or placebo, with a RR of 0.80 (95\% CI 0.63-1.01). However, if the results from the study by van Velzen et al. are added to those from this systematic review, the overall power of the aggregated studies is improved, and shortterm treatment non-response is significantly lower in the antibiotic group than in the placebo group, with a RR of $0.77(0.63-0.94 ; p=0.01)^{64}$.

Sputum purulence has been highlighted as a biomarker that may predict better response to antibiotic therapy for AECOPDs ${ }^{65}$. Although no difference in treatment non-response was noted in the study by van Velzen et al. between patients with and without sputum purulence, the study was also probably underpowered to examine this subgroup. Therefore, although findings from this trial suggest that antibiotics (doxycline) added to oral steroids for treatment of outpatient AECOPD do not prolong time to next exacerbation, the trial findings actually lend support to the role of antibiotics in hastening symptom resolution, especially when analysed in the context of results from past clinical trials ${ }^{64}$.

A recently published American Thoracis Society (ATS)/European Respiratory Society (ERS) guideline on management of AECOPD conducted a pooled analysis of several trials and found that antibiotic therapy decreased treatment failure (27.9\% versus $42.2 \%$; RR 0.67, $95 \%$ CI $0.51-0.87)^{66}$. The guideline also found that antibiotics prolonged the time to the next exacerbation (difference of medians 73 days, $\mathrm{p}=0.015$ ). The guideline authors concluded that antibiotic therapy reduced the risk of treatment failure and increased the time between AECOPD. However, the guideline noted that $58 \%$ of patients in the placebo group avoided treatment failure, suggesting that not all exacerbations require treatment with antibiotics. Identifying sensitive and specific biomarkers of bacterial infection (such as pro-calcitonin, or others) may eventually allow 
more precise determination of which patients with exacerbations will benefit from antibiotic therapy.

\section{CONCLUSIONS}

This review has described the current state of the art with respect to how we define, predict, and treat COPD exacerbations. Several controversies and challenges associated with optimal diagnosis and management of exacerbations have been discussed. The current diagnostic criteria for COPD exacerbations are non-specific and need to be refined, as suggested in this review. Hopefully in the future, phenotyping of AECOPD can be advanced, and biomarkers can be discovered, so that the aetiology, and subsequent early treatment of AECOPD can be optimised and personalised. In the meantime, a review of the literature suggests that an integrated COPD self-management strategy comprised of patient education, an action plan, and access to a case manager may lead to earlier therapy for exacerbations and that this strategy decreases respiratory-related hospitalizations. Outpatient therapy for exacerbations should include empiric antibiotic therapy, since antibiotics can reduce the risk of treatment failure.

\section{DISCLOSURES}

Dr. Aaron has nothing to disclose.

\section{REFERENCES}

1. Seemungal TA, Donaldson GC, Paul EA. Effect of exacerbation on quality of life in patients with chronic obstructive pulmonary disease. Am J Respir Crit Care Med. 1998;157:1418-22.
2. Aaron SD. Management and prevention of exacerbations of COPD. BMJ. 2014;349.

3. Kanner RE, Anthonisen NR, Connett JE. Lower respiratory illnesses promote FEV(1) decline in current smokers but not ex-smokers with mild chronic obstructive pulmonary disease: results from the lung health study. Am J Respir Crit Care Med. 2001;164:358-64.

4. Groenewegen KH, Schols AMWJ, Wouters EFM. Mortality and Mortality-Related Factors After Hospitalization for Acute Exacerbation of COPD*. Chest. 2003;124:459-67.

5. Dransfield MT, Kunisaki KM, Strand MJ et al. Acute Exacerbations and Lung Function Loss in Smokers with and without Chronic Obstructive Pulmonary Disease. American Journal of Respiratory and Critical Care Medicine. 2017;195:324-30.

6. Aaron S, Vandemheen K, Ramsay T et al. Multi analyte profiling and variability of inflammatory markers in blood and induced sputum in patients with stable COPD. Respiratory Research. 2010;11:41.

7. Aaron SD, Vandemheen K, Clinch J et al. Measurement of short-term changes in dyspnea and disease-specific quality of life following an acute COPD exacerbation. Chest. 2002;121:688-96.

8. Han MK, Quibrera PM, Carretta EE et al. Frequency of exacerbations in patients with chronic obstructive pulmonary disease: an analysis of the SPIROMICS cohort. The Lancet Respiratory Medicine. 2017;5:619-26.

9. Sadatsafavi M, Xie H, Etminan M, Johnson K, FitzGerald JM, for the Canadian Respiratory Research N. The association between previous and future severe exacerbations of chronic obstructive pulmonary disease: Updating the literature using robust statistical methodology. PLOS ONE. 2018;13: e0191243.

10. Global Strategy for the Diagnosis, Management, and Prevention of Chronic Obstructive Pulmonary Disease. Available from: https://goldcopd.org/ wp-content/uploads/2018/11/GOLD-2019-v1.7-FINAL-14Nov2018-WMS. pdf. Accessed on 28 April 2019.

11. Vogelmeier CF, Criner GJ, Martinez FJ et al. Global Strategy for the Diagnosis, Management, and Prevention of Chronic Obstructive Lung Disease 2017 Report. GOLD Executive Summary. Am J Respir Crit Care Med. 2017; 195:557-82.

12. Beghe B, Verduri A, Roca M, Fabbri LM. Exacerbation of respiratory symptoms in COPD patients may not be exacerbations of COPD. Eur Respir J. 2013;41:993-5.

13. Rodriguez-Roisin R. Toward a consensus definition of COPD exacerbations. Chest. 2000;117:398S-401S.

14. Quint JK, Donaldson GC, Hurst JR, Goldring JJP, Seemungal TR, Wedzicha JA. Predictive accuracy of patient-reported exacerbation frequency in COPD. Eur Respir J. 2011;37:501-7.

15. Leidy NK, Wilcox TK, Jones PW, Roberts L, Powers JH, Sethi S. Standardizing Measurement of Chronic Obstructive Pulmonary Disease Exacerbations. Am J Respir Crit Care Med. 2011;183:323-9.

16. Leidy N, Murray L. Patient-reported Outcome (PRO) Measures for Clinical Trials of COPD: The EXACT and E-RS. COPD. 2013;10:393-8.

17. Langsetmo L, Platt RW, Ernst P, Bourbeau J. Underreporting Exacerbation of Chronic Obstructive Pulmonary Disease in a Longitudinal Cohort. Am J Respir Crit Care Med. 2008;177:396-401.

18. Vijayasaratha K, Stockley RA. Reported and Unreported Exacerbations of COPD: Analysis by Diary Cards. Chest. 2008;133:34-41.

19. Wolpin S, Nguyen HQ, Donesky-Cuenco D, Carrieri-Kohlman V, Doorenbos A. Effects of Automated Prompts for Logging Symptom and Exercise Data on Mobile Devices in Patients With Chronic Obstructive Pulmonary Disease. CIN: Computers, Informatics, Nursing. 2011;29:TC3-TC8.

20. Najafzadeh M, Marra CA, Sadatsafavi M et al. Cost effectiveness of therapy with combinations of long acting bronchodilators and inhaled steroids for treatment of COPD. Thorax. 2008;63:962-7.

21. Hunter LC, Lee RJ, Butcher I et al. Patient characteristics associated with risk of first hospital admission and readmission for acute exacerbation of chronic obstructive pulmonary disease (COPD) following primary care COPD diagnosis: a cohort study using linked electronic patient records. BMJ Open. 2016;6. 
22. Agusti A, Faner R, Celli B, Rodriguez-Roisin R. Precision medicine in COPD exacerbations. Lancet Respir Med. 2018;6:657-9.

23. Celli BR, Barnes PJ. Exacerbations of chronic obstructive pulmonary disease. Eur Respir J. 2007;29:1224-38.

24. Bafadhel M, McKenna S, Terry S et al. Acute Exacerbations of Chronic Obstructive Pulmonary Disease: Identification of Biologic Clusters and Their Biomarkers. Am J Respir Crit Care Med. 2012;184:662-71.

25. Noell G, Cosío BG, Faner R et al. Multi-level differential network analysis of COPD exacerbations. Eur Respir J. 2017;50.

26. Donaldson GC, Seemungal TAR, Patel IS et al. Airway and Systemic Inflammation and Decline in Lung Function in Patient with COPD. Chest. 2005; 128:1995-2004.

27. Aaron SD, Angel JB, Lunau M et al. Granulocyte Inflammatory Markers and Airway Infection during Acute Exacerbation of Chronic Obstructive Pulmonary Disease. Am J Respir Crit Care Med. 2001;163:349-55.

28. Bafadhel M, McKenna S, Terry S et al. Blood Eosinophils to Direct Corticosteroid Treatment of Exacerbations of Chronic Obstructive Pulmonary Disease. A Randomized, Placebo-Controlled Trial. Am J Respir Crit Care Med. 2012;186:48-55.

29. O'Donnell DE, Parker CM. COPD exacerbations · 3: Pathophysiology. Thorax. 2006;61:354-61.

30. Parker CM, Voduc N, Aaron SD, Webb KA, O'Donnell DE.. Physiological changes during symptom recovery from moderate exacerbations of COPD. Eur Respir J. 2005;26:420-8.

31. Sethi S, Evans N, Brydon JB, Murphy TF. New strains of bacteria and exacerbations of chronic obstructive pulmonary disease. N Eng J Med. 2002;347:465-71.

32. Sethi S, Murphy TF. Infection in the pathogenesis and course of chronic obstructive pulmonary disease. N Engl J Med. 2008;359:2355-65.

33. Seemungal TAR, Harper-Owen R, Bhowmik A et al. Respiratory viruses, symptoms, and inflammatory markers in acute exacerbations and stable chronic obstructive pulmonary disease. Am J Resp Crit Care Med. 2001;164:1618-23.

34. Hewitt R, Farne H, Ritchie A, Luke E, Johnston SL, Mallia P. The role of viral infections in exacerbations of chronic obstructive pulmonary disease and asthma. Ther Adv Respir Dis. 2016;10:158-74.

35. Murphy TF, Brauer AL, Grant BJB, Sethi S. Moraxella catarrhalis in Chronic Obstructive Pulmonary Disease. Am J Respir Crit Care Med. 2005;172:195-9.

36. Wilkinson TMA, Hurst JR, Perera WR, Wilks M, Donaldson GC, Wedzicha JA. Effect of interactions between lower airway bacterial and rhinoviral infection in exacerbations of COPD. CHEST. 2006;129:317-24.

37. Molyneaux PL, Mallia P, Cox MJ et al. Outgrowth of the Bacterial Airway Microbiome after Rhinovirus Exacerbation of Chronic Obstructive Pulmonary Disease. Am J Respir Crit Care Med. 2013;188:1224-31.

38. Papi A, Bellettato CM, Braccioni F et al. Infections and Airway Inflammation in Chronic Obstructive Pulmonary Disease Severe Exacerbations. Am J Respir Crit Care Med. 2006;173:1114-21.

39. Sapey E, Stockley RA. COPD exacerbations. 2: Aetiology. Thorax. 2006;61:250-8.

40. Peacock JL, Anderson HR, Bremner SA, et al. Outdoor air pollution and respiratory health in patients with COPD. Thorax 2011;66:591-596.

41. Stolz D, Christ-Crain M, Bingisser R et al. Antibiotic treatment of exacerbations of COPD: a randomized, controlled trial comparing procalcitonin-guidance with standard therapy. Chest. 2007;131:9-19.

42. Karakioulaki M, Stolz D. The Case of Procalcitonin for Lower Respiratory Tract Infections. BRN Rev. 2019;5:277-93.

43. Mathioudakis AG, Chatzimavridou-Grigoriadou V, Corlateanu A, Vestbo J. Procalcitonin to guide antibiotic administration in COPD exacerbations: a meta-analysis. Eur Respir Rev. 2017;26.

44. Shafiek H, Fiorentino F, Merino JL et al. Using the Electronic Nose to Identify Airway Infection during COPD Exacerbations. PloS one. 2015;10:e135199-e.

45. Hurst JR, Vestbo J, Anzueto A. Susceptibility to exacerbation in chronic obstructive pulmonary disease. N Engl J Med. 2010;363:1128-38.

46. Hurst JR, Donaldson GC, Quint J, Goldring J, Baghai-Ravary R, Wedzicha JA. Temporal Clustering of Exacerbations in Chronic Obstructive Pulmonary Disease. Am J Resp Crit Care Med. 2009; 179:369-74.
47. Han MK, Kazerooni EA, Lynch DA et al. Chronic Obstructive Pulmonary Disease Exacerbations in the COPDGene Study: Associated Radiologic Phenotypes. Radiology. 2011;261:274-82.

48. Gershon AS, Guan J, Victor JC, Goldstein R, To T. Quantifying Health Services Use for Chronic Obstructive Pulmonary Disease. Am J Respir Crit Care Med. 2013;187:596-601.

49. Wilkinson T, Donaldson GC, Hurst JR, Seemungal TAR, Wedzicha JA Early therapy improves outcomes of exacerbations of chronic obstructive pulmonary disease. Am J Resp Crit Care Med. 2004;169:1298-303.

50. Chandra D, Tsai CL, Camargo CA. Acute Exacerbations of COPD: Delay in Presentation and the Risk of Hospitalization. COPD. 2009;6:95-103.

51. Hurst JR, Donaldson GC, Perera W et al. Use of Plasma Biomarkers at Exacerbation of Chronic Obstructive Pulmonary Disease. Am J Respir Crit Care Med. 2006;174:867-74

52. Calverley P, Pauwels RA, Jones PW, Anderson JA, Vestbo J. The severity of airways obstruction as a determinant of treatment response in COPD. Int J Chron Obstruct Pulmon Dis. 2006;1:209-18.

53. Seemungal TA, Donaldson GC, Bhowmik A. Time course and recovery of exacerbations in patients with chronic obstructive pulmonary disease. Am J Respir Crit Care Med. 2000;161:1608-13

54. Aaron SD, Donaldson GC, Whitmore GA, Hurst JR, Ramsay T, Wedzicha JA. Time course and pattern of COPD exacerbation onset. Thorax. 2012;67: 238-43.

55. Lenferink A, Brusse-Keizer M, van der Valk PD et al. Self-management interventions including action plans for exacerbations versus usual care in patients with chronic obstructive pulmonary disease. Cochrane Database Syst Rev. 2017;8:CD011682-CD.

56. Zwerink M, Brusse-Keizer M, van der Valk P et al. Self management for patients with chronic obstructive pulmonary disease. Cochrane Database Syst Rev. 2014(3).

57. Fan V, Gaziano, JM, Lew R et al. A Comprehensive Care Management Program to Prevent Chronic Obstructive Pulmonary Disease Hospitalizations. Ann Intern Med. 2012;156:I-30.

58. Nici L, Bontly TD, ZuWallack R, Gross N. Self-Management in Chronic Obstructive Pulmonary Disease. Time for a Paradigm Shift? Ann Amer Thorac Soc. 2014;11:101-7.

59. Bourbeau J, Julien M, Maltais F et al. Reduction of hospital utilization in patients with chronic obstructive pulmonary disease: a disease-specific self-management intervention. Arch Intern Med. 2003;163:585-91.

60. Rice KL, Dewan N, Bloomfield HE et al. Disease Management Program for Chronic Obstructive Pulmonary Disease. Am J Respir Crit Care Med. 2010 182:890-6.

61. Anthonisen NR, Manfreda J, Warren CP, Hershfield ES, Harding GK, Nelson NA. Antibiotic therapy in exacerbations of chronic obstructive pulmonary disease. Ann Intern Med. 1987;106:196-204.

62. Llor C, Moragas A, Hern+índez S, Bayona C, Miravitlles M. Efficacy of Antibiotic Therapy for Acute Exacerbations of Mild to Moderate Chronic Obstructive Pulmonary Disease. Am J Respir Crit Care Med. 2012;186: 716-23.

63. van Velzen P, ter Riet G, Bresser P et al. Doxycycline for outpatient-treated acute exacerbations of COPD: a randomised double-blind placebo-controlled trial. Lancet Respir Med. 2017;5:492-9.

64. Labaki WW, Han MK. Antibiotics for COPD exacerbations. Lancet Respir Med. 2017;5:461-2

65. Aggarwal D, Mohapatra PR, Aggarwal P. Significance of sputum purulence to guide antibiotic therapy in exacerbations of COPD. Eur Respir J. 2013, 41:248.

66. Wedzicha JA, Miravitlles M, Hurst JR et al. Management of COPD exacerbations: a European Respiratory Society/American Thoracic Society guideline. Eur Respir J. 2017;49:1600791.

67. Han MK, Martinez FJ. Pharmacotherapeutic Approaches to Preventing Acute Exacerbations of Chronic Obstructive Pulmonary Disease. Proc Am Thor Soc. 2011;8:356-62. 